\title{
Clinical Application of Video Head Impulse Test in Acute Vestibular Syndrome
}

\author{
Myung Hoon Yoo \\ Department of Otorhinolaryngology-Head and Neck Surgery, School of Medicine, Kyungpook National University, Daegu, Korea
}

급성 전정 증후군에서 비디오 두부충동검사의 임상적 적용

유명훈

경북대학교 의과대학 이비인후-두경부외과학교실

\author{
Received December 3, 2019 \\ Revised January 7, 2020 \\ Accepted January 13, 2020 \\ Address for correspondence \\ Myung Hoon Yoo, MD, PhD \\ Department of Otorhinolaryngology- \\ Head and Neck Surgery, \\ Kyungpook National University \\ Chilgok Hospital, 807 Hoguk-ro, \\ Buk-gu, Daegu 41404, Korea \\ Tel $+82-53-200-2168$ \\ Fax $+82-53-200-2029$ \\ E-mail cooleo312@gmail.com
}

Acute unilateral vestibulopathy (AUV) is the recommended term [rather than the more widely used 'vestibular neuritis (VN)'] for all pathologies involving sudden impairment of the unilateral peripheral vestibular function regardless of the exact location of the lesion. Acute vestibular syndrome (AVS) is an upper-level entity of AUV, which is the most common cause of AVS, and includes acute central vertigo. AVS typically presents with the symptoms of new onset continuous vertigo, gait instability, and nausea/vomiting lasting several days to weeks. The video head impulse test (vHIT) was recently developed and has been widely adopted at clinics and emergency centers. In this study, we will review the differential diagnosis of AVS using vHIT. In addition, we will describe the subtypes of VN and the use of vHIT for follow-up testing in AVS. Korean J Otorhinolaryngol-Head Neck Surg 2020;63(1):3-13

Key Words Acute unilateral vestibulopathy · Acute vestibular syndrome · Vestibular neuritis · Video head impulse test.

\section{Introduction}

Vestibular neuritis (VN) presents as an acute spontaneous whirling-type dizziness caused by sudden unilateral vestibular dysfunction. ${ }^{1)}$ However, labyrinthitis, sudden hearing loss with dizziness, and neurolabyrinthitis may present similar symptoms and signs as VN. Therefore, the term acute unilateral vestibulopathy (AUV) is recommended instead of VN to define all pathologies involving a sudden decrease of unilateral peripheral vestibular function regardless of the exact location of the lesion. On the other hand, acute vestibular syndrome (AVS) is an upper-level entity that includes both AUV, which is the most common cause of AVS (up to 70\%), and acute central vertigo [usually posterior circulation stroke (PCS)].

This is an Open Access article distributed under the terms of the Creative Commons Attribution Non-Commercial License (https://creativecommons.org/licenses/by-nc/4.0) which permits unrestricted non-commercial use, distribution, and reproduction in any medium, provided the original work is properly cited.
AVS typically presents with the symptoms of new onset continuous vertigo, motion intolerance, gait instability, and nausea/vomiting lasting several days to weeks. ${ }^{2-5)}$

The Head Impulse, Nystagmus, and Test of Skew (HINTS) battery test [including a head impulse test (HIT), examination of gaze-evoked nystagmus, and test of skew deviation] is well known as an easily available bedside examination that can better differentiate AUV from central vertigo at the early stage compared to diffusion weighted MRI. ${ }^{6}$ Among the tests included in the HINTS battery, horizontal HIT has been established in previous reports as a simple and useful test for evaluating the vestibule-ocular reflex (VOR) with high sensitivity.

The recent development and application of a video head impulse test (vHIT) device have led to great improvements in the differential diagnosis and treatment of AVS for patients with dizziness at both clinics and emergency centers. The de- 
vice has several advantages over bedside HIT including the ability to record saccades, calculate gains, observe covert saccades, and test the vertical canals. Compared to the caloric test, which is the golden standard test for evaluating VOR, vHIT is less uncomfortable for the patient and requires a shorter period of time. ${ }^{4,5,7)}$ Therefore, the test can be easily performed as a first vestibular function test at clinics for dizzy patients. Our aim is to review the effectiveness of vHIT for the clinical diagnosis and treatment of AVS.

\section{Differential diagnosis}

AVS, which refers to a higher level concept than AUV, includes both central and peripheral dizziness. Although the most common cause of AVS is VN, PCS may present similar symptoms and signs as VN. In clinics, the simple examinations included in the HINTS battery are more sensitive than early MRI. ${ }^{6,8)}$ Among the three conditions tested in HINTS examinations, mild skew deviation is rare, but when detected, it strongly suggests a central lesion. Gaze-evoked nystagmus is also observed in only about $50 \%$ of cerebellar strokes, but strongly suggests a central problem. HIT is the most important test for central and peripheral differentiation, suggesting a central lesion when negative. A corrective saccade in the vHIT strongly suggests a peripheral lesion, but anterior inferior cerebellar artery (AICA) infarction may also show abnormal vHIT. If other neurologic signs such as hearing loss, facial palsy, ataxia, or sensory deficit are accompanied with abnormal vHIT, than AICA infarction should be suspected in patients with high risk factors of infarction. Therefore, the diagnosis should be confirmed using other neurological examinations. $^{4,8,10)}$

A bedside HIT, directly observed with the eyes, has several disadvantages: it is difficult to observe the covert saccades that occur when the head moves, it is highly dependent on the proficiency of the examiner; and it is difficult to evaluate quantitatively. The vHIT can be used in emergency departments or outpatient clinics as an alternative to compensate for these disadvantages for the differentiation of AVS. ${ }^{11,12)}$ The gain of the vHIT can be used to differentiate VN from PCS with $88 \%$ sensitivity and $92 \%$ specificity, which is more accurate than early diffusion-weighted image MRI. ${ }^{10,13)}$ According to Mantokoudis, et al., ${ }^{14)}$ a lateral semicircular canal gain over 0.7041 was reported as an optimal discrimination cut-off point for stroke. In cases of PCS infarction, when the inner ear is involved in AICA infarction, gain reduction can be observed on the lesion side, but if the vestibular nucleus is damaged, symmetrical gain reduction with small catch-up saccades can be seen on both sides. In cases of posterior inferior cerebellar artery (PICA) infarction, there is a slight symmetrical gain reduction on both sides and small catch-up saccades (Fig. 1, Table 1). ${ }^{12)}$ However, it has been reported that the gain of vHIT can vary in cases of anterior cerebellar infarction, thus, differential diagnosis only based on the gain of vHIT can be risky. Clinicians must also consider AICA infarctions during differential diagnosis despite positive results in bilateral vHIT, as they are not always attributable to peripheral lesions. ${ }^{11,12,15)}$

In addition, unlike bedside HIT, which is directly seen with the eyes, artifacts can occur during vHIT testing (like slippage of the goggles); therefore, clinicians should avoid mistakes that can occur when performing a diagnosis based on the normal range of the gain provided by the vHIT. ${ }^{3)}$ The value of the gain can significantly vary according to the algorithms and the type of vHIT device used. ${ }^{16)}$ In addition to the absolute value of the gain, the canal function can also be determined using bilateral gain asymmetry, as in cases of canal paresis determined with the bithermal caloric test: gain asymmetry $(\mathrm{GA})=($ right gain - left gain $) /($ right gain + left gain $) \times 100 \%$. The normal range of GA has not been established yet, but it can be considered abnormal if it is $8 \%$ or greater regardless of age. $^{17-19)}$ Furthermore, even if the gain is normal, it is also important to differentiate abnormal saccades, since they can appear in normal function and can have similar shapes in spontaneous nystagmus and eye blinking. ${ }^{20)}$ According to a previous report, when the peak velocity of the corrective saccade exceeds $100 \%$ and when the peak velocity difference between the ears is more than $40 \%$, the saccadic movement can be defined as abnormal. ${ }^{17,18)}$ However, if the peak velocity criteria are used for the determination of abnormality, the given head impulse velocity is very important and should not be too high or too low (typically $150-250 \%$ s).

When a patient visits with acute vertigo symptoms consistent with $\mathrm{VN}$ and unilateral sudden onset hearing loss for the 1st attack presentation, the clinical diagnosis could be 'sudden hearing loss with vertigo' or 'labyrinthitis' after the exclusion of AICA infarction. In a previous study using vHIT on patients with sudden hearing loss with vertigo, vHIT showed abnormalities most often in the posterior semicircular canal ( $74 \%$ of patients), and the frequency was much higher than the $41 \%$ observed in the lateral canal and the $30 \%$ observed in the superior canal. The involvement of the posterior canal in sudden hearing loss with vertigo has a different pattern from that of typical VN and is thought to be caused by focal 

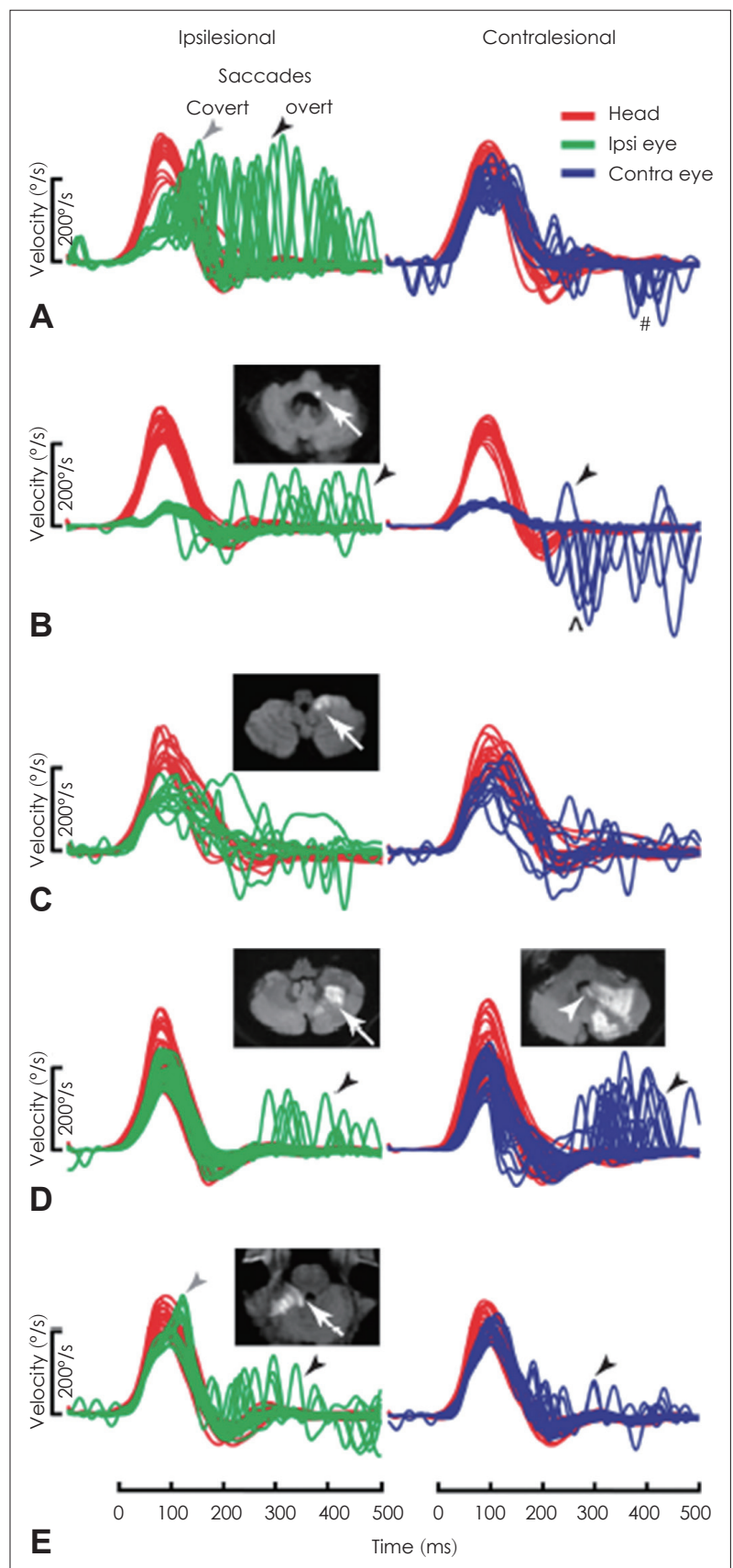

Fig. 1. In vestibular neuritis (VN), the ipsilateral gain is decreased and shows large overt saccades (black arrowhead) with frequent covert saccades (gray arrowhead), while the contralateral gain is slightly decreased and shows small saccades (A). In anterior inferior cerebellar artery-peripheral (AICAp) infarction (white arrow showing infarction site), the gains on both sides decrease, but the ipsilateral saccade is smaller than that observed in $\mathrm{VN}$ and the contralateral saccade is larger than that in VN (B). In anterior inferior cerebellar artery-central (AICAc) infarction due to isolated right floccular infarction, both gains are mildly decreased and some small saccades are visible (C). In posterior inferior cerebellar artery (PICA) infarction involving the left cerebellar hemisphere and nodulus (white arrowhead) (D), the gain is symmetrical and overt saccades are frequent. In superior cerebellar artery (SCA) infarction, both gains are slightly decreased and also show small overt saccades (E). Adapted from: Halmagyi, et al. Front Neurol 2017; 9(8):258. ${ }^{12)}$ infarction of the common cochlear artery, which supplies the cochlea and posterior semicircular canal. ${ }^{3,21,22)}$ In a study evaluating the recovery of vestibular function in patients with Ramsay-Hunt syndrome with vertigo and those with VN using vHIT, patients with VN recovered significantly faster than those with Ramsay-Hunt syndrome. ${ }^{23)}$

\section{Subtypes of VN}

As mentioned earlier, rather than evaluating abnormalities in the lateral semicircular canal based on catch-up saccades with a bedside HIT, quantitative evaluation of semicircular canal functions are now possible using vHIT as well as the evaluation of each vertical canal. In addition, the subtypes of VN can be distinguished using the cervical vestibular-evoked myogenic potential (cVEMP) that measures the function of the saccule (inferior vestibular nerve), ocular vestibular myogenic potential (oVEMP) that measures the function of the utricle (superior vestibular nerve), subjective visual vertical test, and fundus photography (Fig. 2). ${ }^{24-26)}$ There have also been reports of different types of AUV that invade only one canal or one vestibular organ in the superior or inferior vestibular nerve branch. This condition may also be called ampullary $\mathrm{VN}^{20,24,27)}$

\section{Superior VN}

The most common ( $>50 \%)$ type of AUV is superior VN. In general, superior VN shows canal paresis in the caloric test with abnormal findings in oVEMP and normal cVEMP results. In vHIT for superior VN, the gain in the ipsilateral lateral canal and superior canal is reduced and catch-up saccade can be observed (Fig. 3). In the literature, there are a few reports of isolated lateral canal involvement in vHIT reported for VN patients but no reports of isolated superior canal involvement. $^{20,25)}$

\section{Total VN}

Total VN (or pan-vestibular neuritis), involving the superior and inferior vestibular nerves, occurs in about one third of all patients with VN. For patients with total VN, vHIT shows a decrease in gain and saccades in all three canals. ${ }^{20,24,25)}$

\section{Inferior VN}

In inferior $\mathrm{VN}$, a relatively rare $(2 \%$ to $15 \%)$ type of $\mathrm{VN}$, vHIT shows abnormalities in only the posterior semicircular canal with normal findings in the lateral and superior canals (Fig. 4). ${ }^{28)}$ Additionally, vestibular dysfunction is observed on 
Table 1. Differential diagnosis using vHIT in acute vestibular syndrome

\begin{tabular}{|c|c|c|c|}
\hline Disease & VHIT saccade & VHIT gain & Accompanied symptoms and signs \\
\hline $\mathrm{VN}$ & $\begin{array}{l}\text { Large overt and covert saccades } \\
\text { in lesion side/ small overt saccades } \\
\text { in contralateral side }\end{array}$ & $\begin{array}{l}\text { Markedly decreased in lesion } \\
\text { side and mild decrease in } \\
\text { contralateral side }\end{array}$ & Nausea, vomiting \\
\hline AICA infarction & $\begin{array}{l}\text { Overt saccades in both sides (less } \\
\text { asymmetry in saccade amplitude } \\
\text { than } \mathrm{VN} \text { ) }\end{array}$ & $\begin{array}{l}\text { Bilateral gain reduction } \\
\text { (lesser asymmetry than VN) }\end{array}$ & $\begin{array}{l}\text { Hearing loss, facial palsy, sensory } \\
\text { deficit, dysphagia/perverted } \\
\text { nystagmus }\end{array}$ \\
\hline $\mathrm{PICA} / \mathrm{SCA}$ infarction & $\begin{array}{l}\text { Small overt saccades in both sides } \\
\text { (saccades are more frequent and/or } \\
\text { larger in contralateral side than VN) }\end{array}$ & $\begin{array}{l}\text { Symmetric [normal or mild } \\
\text { (up to 20\%) reduction] }\end{array}$ & $\begin{array}{l}\text { Truncal ataxia, headache, facial } \\
\text { pain/perverted nystagmus }\end{array}$ \\
\hline
\end{tabular}

VHIT results of lateral semicircular gain. vHIT: video head impulse test, AICA: anterior inferior cerebellar artery, PICA: posterior inferior cerebellar artery, SCA: superior cerebellar artery, VN: vestibular neuritis

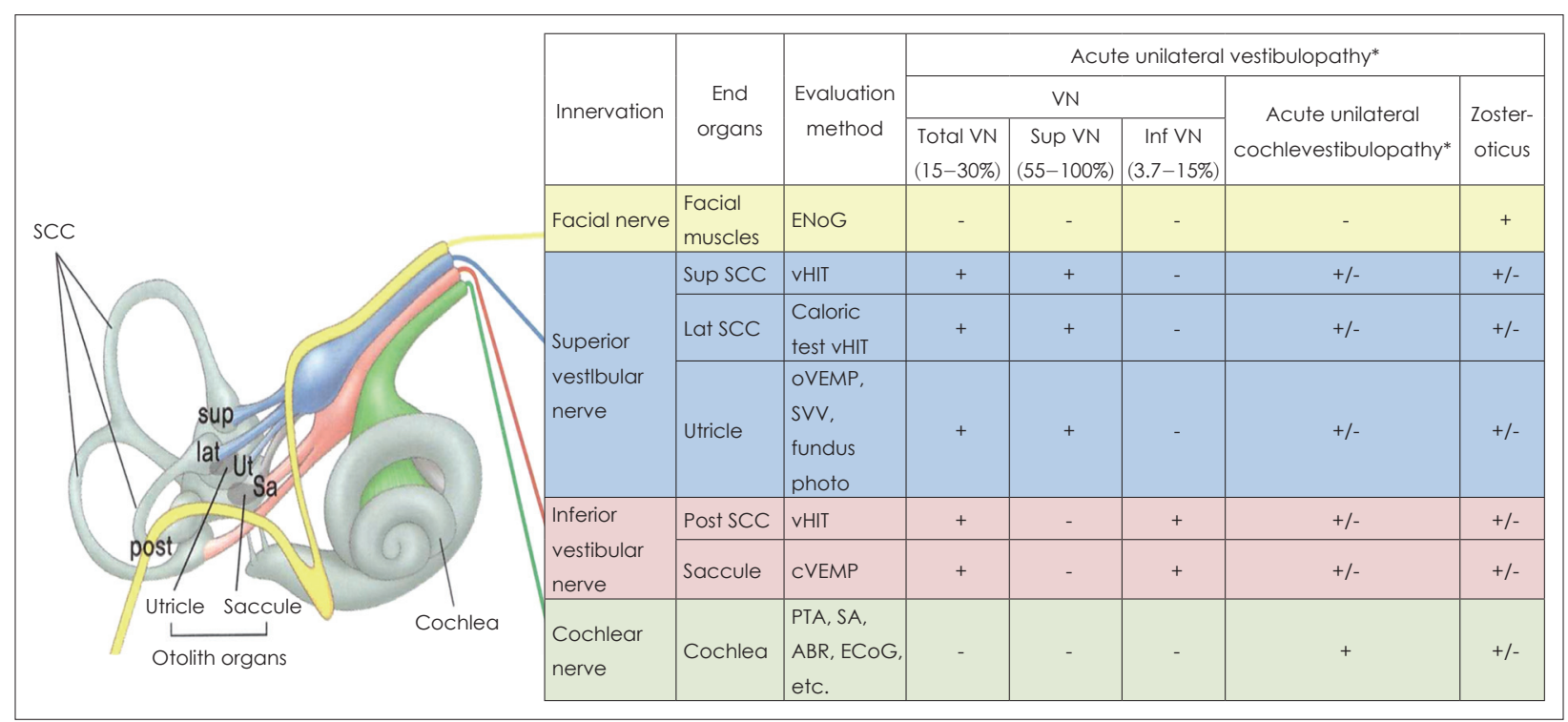

Fig. 2. The involvement of each vestibular end organ can be evaluated by combining various tests. ' + ' indicates an abnormal test result; 'indicates a normal test result; '+/-'indicates both normal and abnormal results can be found; 'small + or -'indicates the corresponding result is less frequent; '*' indicates diagnostic term suggested by International Classificiation of Vestibular Disorders by Barany Society. Adapted from: The Korean Balance Society. Clinical Vestibular Science, with permission of Panmun Education. ${ }^{20)}$ SCC: semicircular canal, Sup: superior, Lat: lateral, Post: posterior, Ut: utricle, Sa: saccule, ENoG: electroneurography, vHIT: video head impulse test, oVEMP: ocular vestibular-evoked myogenic potential, SVV: subjective visual vertical, cVEMP: cervical vestibular-evoked myogenic potential, PTA: pure tone audiometry, SA: speech audiometry, ABR: auditory brainstem reflex, ECoG: electrocochleography, VN: vestibular neuritis, Int: inferior.

the cVEMP. In a typical case of inferior $\mathrm{VN}$, spontaneous nystagmus cannot be seen with a normal caloric function test; hence, this type of VN can be misinterpreted as a central lesion. In this case, vHIT can help distinguish between central and peripheral lesions based on abnormalities in the posterior canal. ${ }^{12,29}$ Recently, in the study by Park, et al. ${ }^{29)}$ on the correlation between oVEMP and the posterior canal of vHIT in inferior $\mathrm{VN}$, the correlation was relatively lower than predicted. This may be because the inferior vestibular nerve is divided into the saccular nerve and posterior ampullary nerve, and thus both tests can be used to complement each other. ${ }^{30}$

\section{Clinical course and follow-up}

The vHIT can be used to evaluate rapid vestibular ocular re- flexes $(2-6 \mathrm{~Hz})$ closer to normal head movements $(0.1-5 \mathrm{~Hz})$ compared to the conventional caloric test $(0.002 \mathrm{~Hz})$ and the rotatory chair test $(0.01-0.64 \mathrm{~Hz}){ }^{31)}$ In addition, the vHIT has a shorter test time than the other tests and causes less inconvenience to the patient. Therefore, it is possible and useful to repeat the test several times for each patient during the follow-up period to validate the treatment results. In a recent study using vHIT and other vestibular function tests to determine the efficacy of steroids in $\mathrm{VN}$, there was no evidence that steroid therapy was effective for the treatment of VN. This finding was in contrast with previous reports using a caloric test that showed the effectiveness of steroids. ${ }^{32}$

The discrepancies between the evaluation of the lateral semicircular canal function using the vHIT and canal paresis 

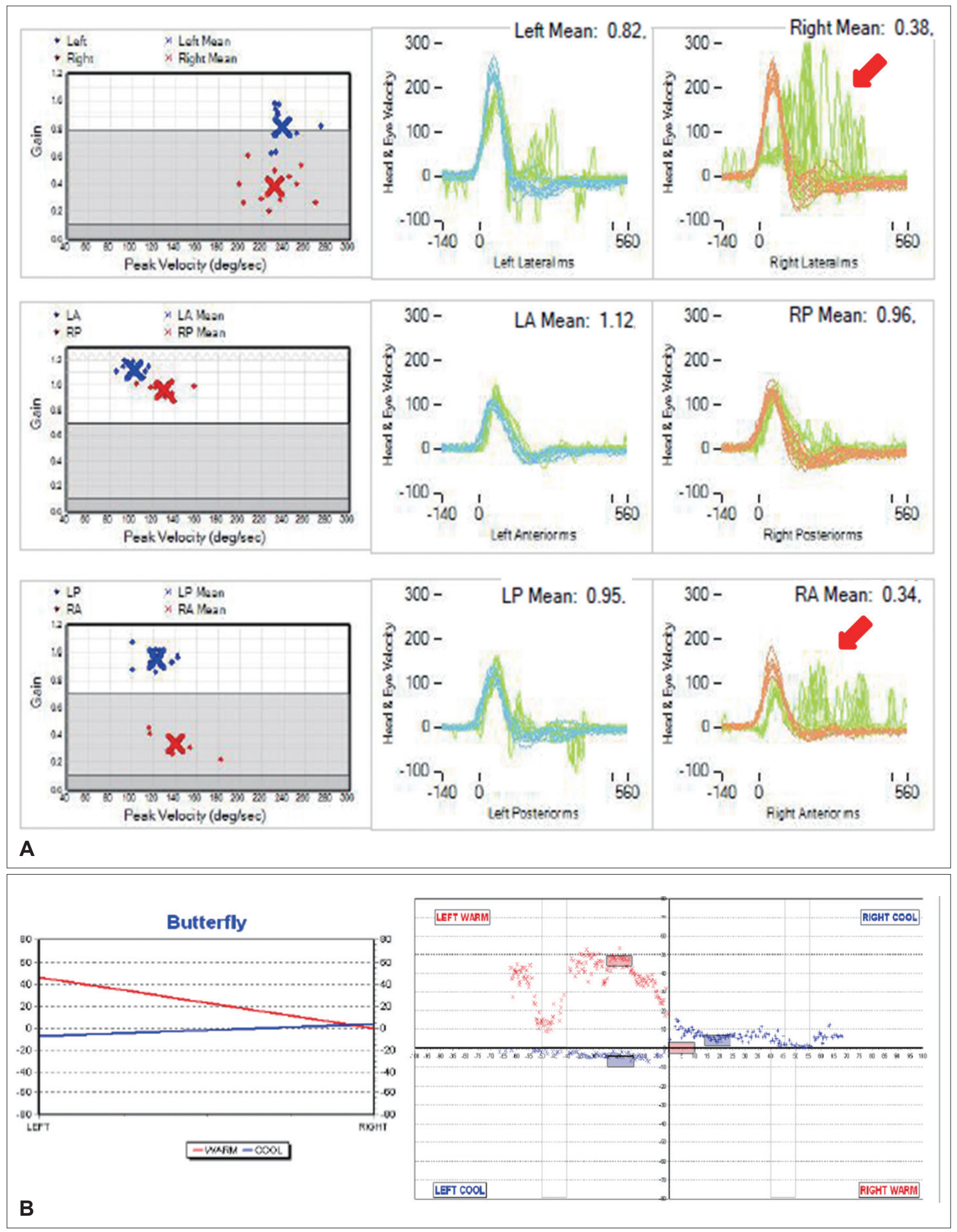

Fig. 3. The results of various vestibular function tests in a 47-year-old male patient with right superior vestibular neuritis (VN). In the video head impulse test (VHIT) (A), right lateral canal dysfunction (gain 0.38) and right superior canal dysfunction (gain 0.34 ) are observed with overt saccades (red arrow). The caloric test (B) shows $86 \%$ canal paresis on the right side. LA: left anterior, LP: left posterior, $\mathrm{RA}$ : right anterior, RP: right posterior. 

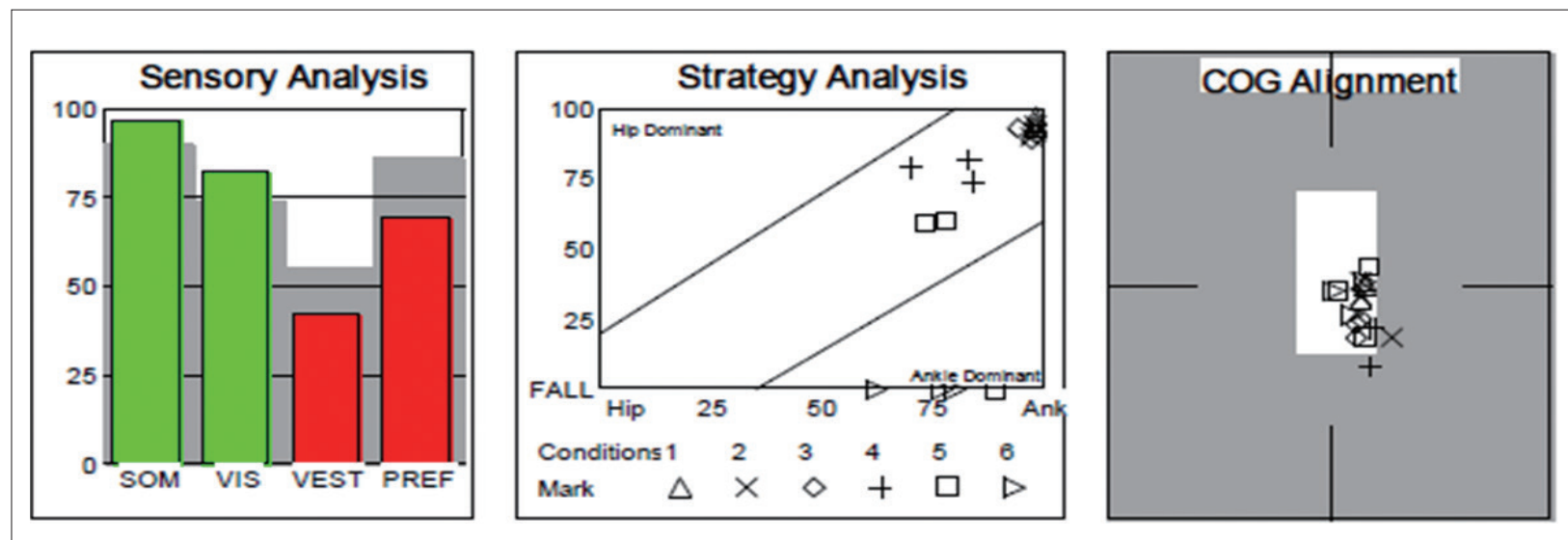

\section{C}

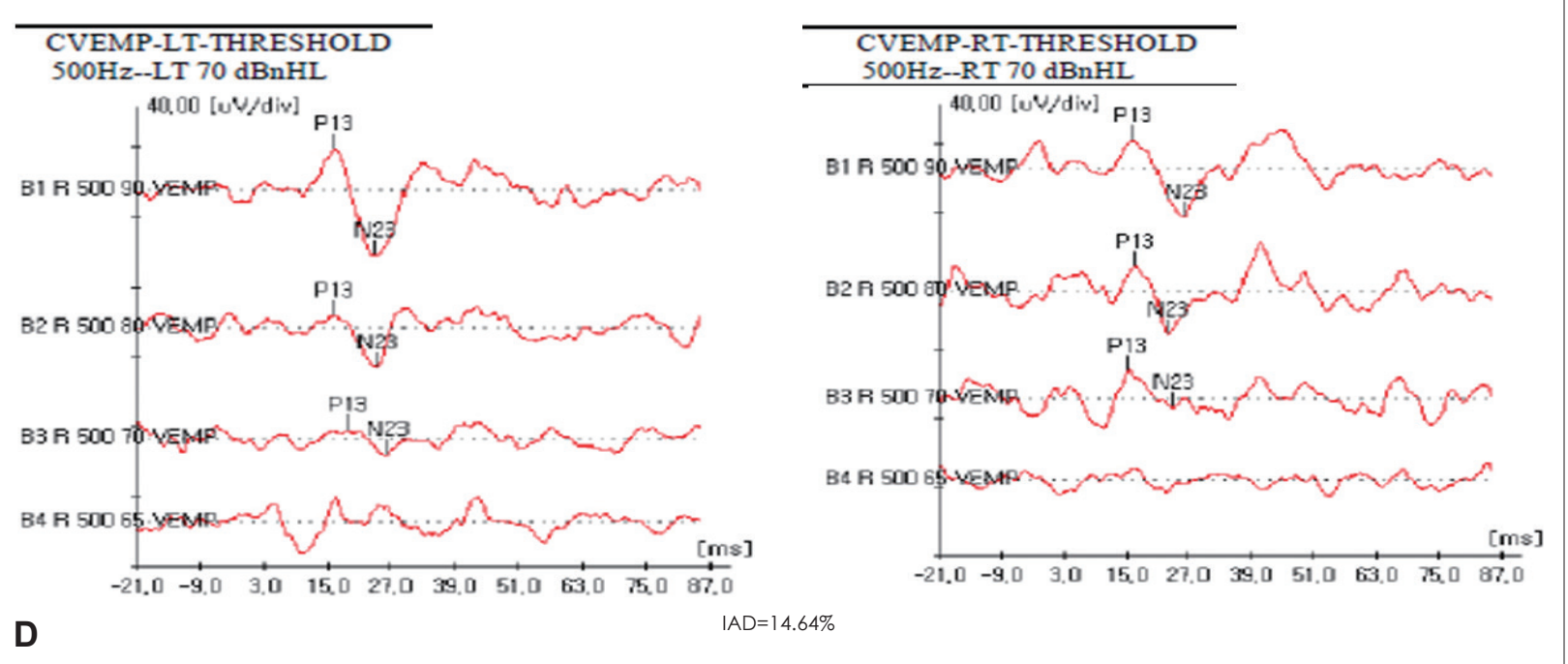

\section{OVEMP-LT-THRESHOLD $500 \mathrm{~Hz}--\mathrm{LT} 75 \mathrm{dBnHL}$}

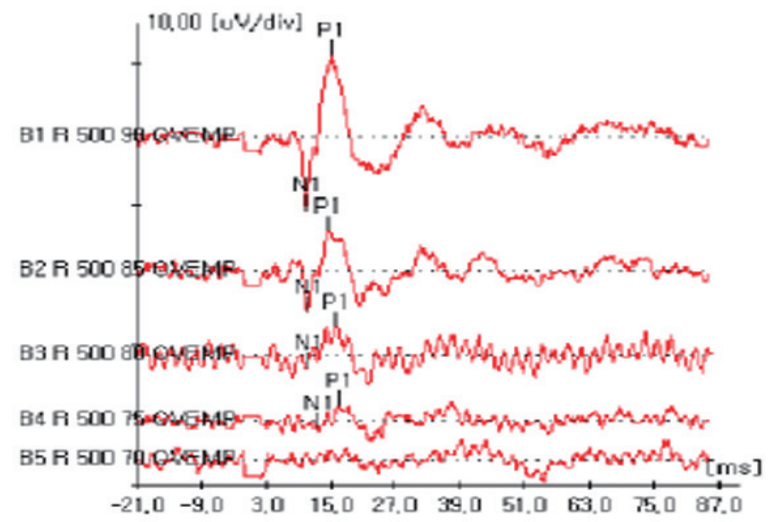

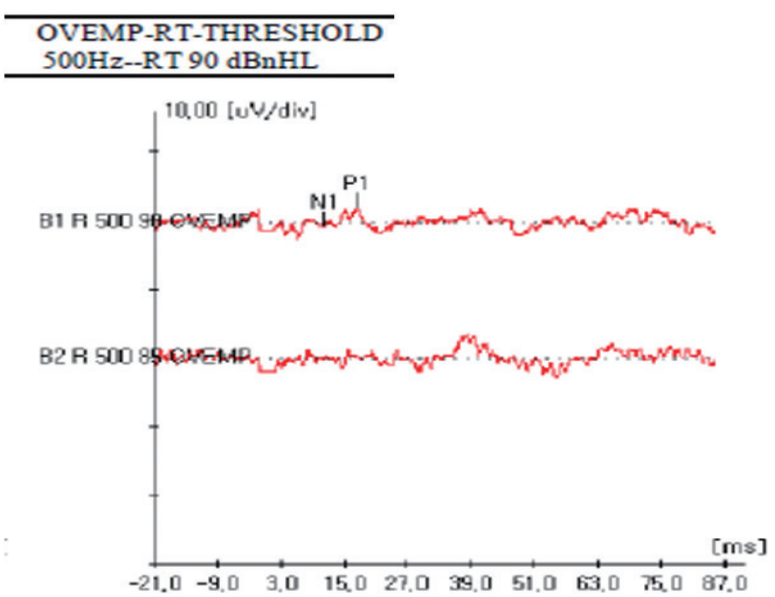

$\mathrm{IAD}=77.82 \%$

E

Fig. 3. The results of various vestibular function tests in a 47-year-old male patient with right superior vestibular neuritis (VN). Sensory organization test of posturography $(C)$ shows mild vestibular dysfunction with vision preference. Cervical vestibular-evoked myogenic potential (VEMP) (D) shows normal results, but ocular VEMP (E) shows abnormal results with an elevated threshold (90 dB) on the right side and greater interaural difference (IAD). By combining these results (involvement of the superior canal, lateral canal, and utricle without the involvement of the posterior canal and saccule), superior VN could be diagnosed. SOM: somatosensory, VIS: visual, VEST: vestibular, PREF: visual preference, COG: center of gravity. 
in the caloric test are relatively well known, especially in Meniere's disease. The discrepancies in Meniere's disease are explained by two theories; first, as we know, there are type I and type II hair cells, and type II hair cells (which usually does not response to high frequency stimulus) are more frequently affected in Meniere's disease, so vHIT can be frequently pre-

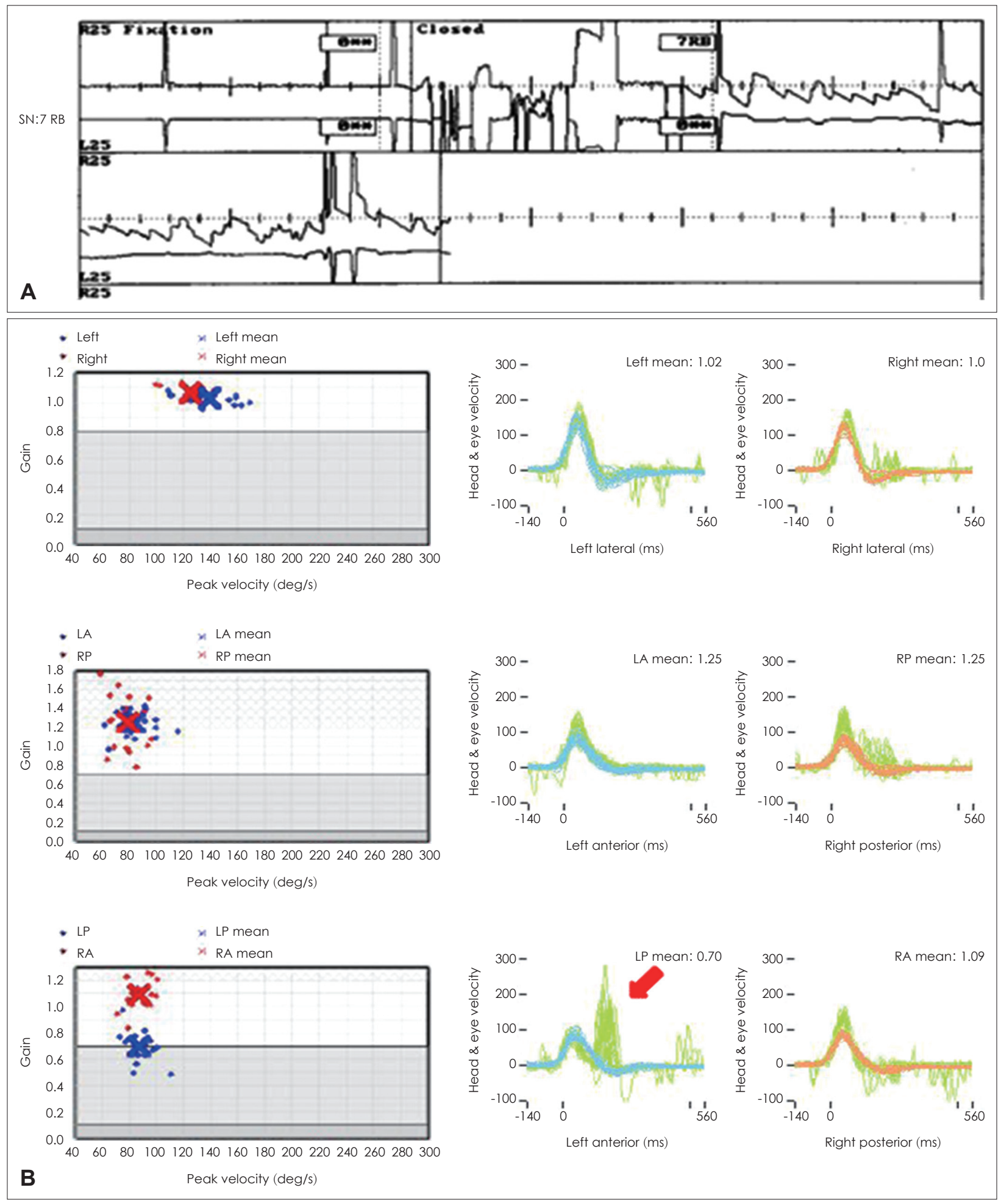

Fig. 4. Video head impulse test (VHIT) result for 45-year-old female patient who visited the clinic for whirling-type vertigo. Spontaneous nystagmus (SN) was found on the right side (7 RB) (A) and vHIT (B) showed large covert saccades (red arrow) in the left posterior canal with borderline gain (0.7). Other canals showed normal gain and the patient was diagnosed with inferior vestibular neuritis. 
served in Meniere's disease (cell type theory); second is 'hydropic labyrinth theory,' physical enlargement of the membranous labyrinth in Meniere's disease would allow heated endolymph circulate within the canal instead of generating a thermally induced pressure across the cupula. Thus, the cupula deflection will be diminished, so reducing the response

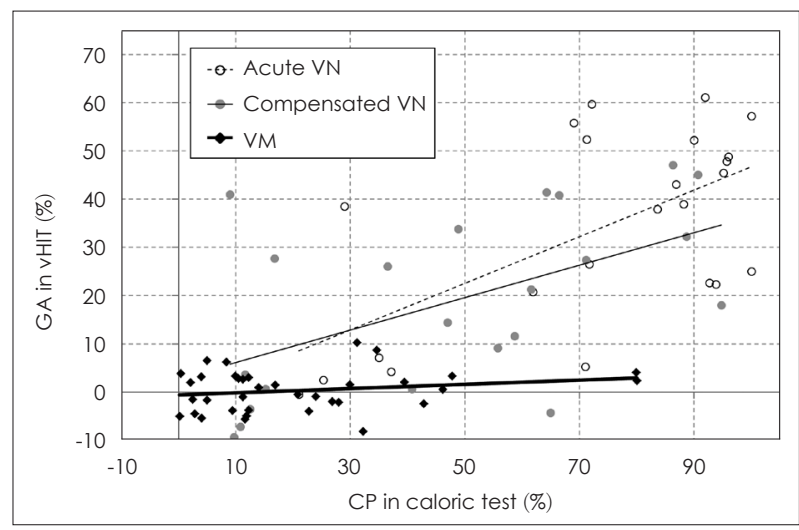

Fig. 5. Correlation between gain asymmetry (GA) of video head impulse test (VHIT) and canal paresis (CP) of caloric test in acute vestibular neuritis (VN), compensated VN (6 months after onset), and vestibular migraine (VM). In VN, there are significant correlations between GA and $\mathrm{CP}$ in both the acute and compensated stages, but the correlation is not significant in VM. Adapted from: Yoo MH, et al. Clin Otolaryngol 2016;41(6):813-7. ${ }^{19)}$ to caloric stimulation. ${ }^{7,20,33-35)}$ However, compared to Meniere's disease, there is a relatively significant correlation between the two tests in $\mathrm{VN}{ }^{35)}$ In a study involving patients with vestibular migraine and patients with VN, the GA of vHIT was significantly correlated with canal paresis in acute $\mathrm{VN}$ $(\mathrm{R}=0.35, p<0.005)$ as well as in chronic $\mathrm{VN}(\mathrm{R}=0.31, p<0.01)$, although there was no significant correlation in the patients with vestibular migraine (Fig. 5). ${ }^{19)}$ Since there is no proven pathophysiology of vestibular migraine and diagnosis is done based on symptoms, this disagreements between two tests in vestibular migraine are difficult to explain but it is presumed that there are frequency-dependent vestibular dysfunction in vestibular migraine resulting different vHIT and caloric results. ${ }^{35,37)}$ So both vHIT and caloric test should be combined to evaluate patient's semicircular canal function for broader spectrum of stimulating frequencies.

In the clinical course of $\mathrm{VN}$, the normalization rate of the caloric test is known to be $40-66 \%$ after 6 months to 1 year. ${ }^{17,23,32,34,38,39)}$ In the case of vHIT, the normalization rate was reported to be higher than that of the caloric test. ${ }^{17)}$ In other studies, the normalization rate of the lateral canal gain in the lesion was $38-52 \%$ at 1 month after onset and $79-87 \%$

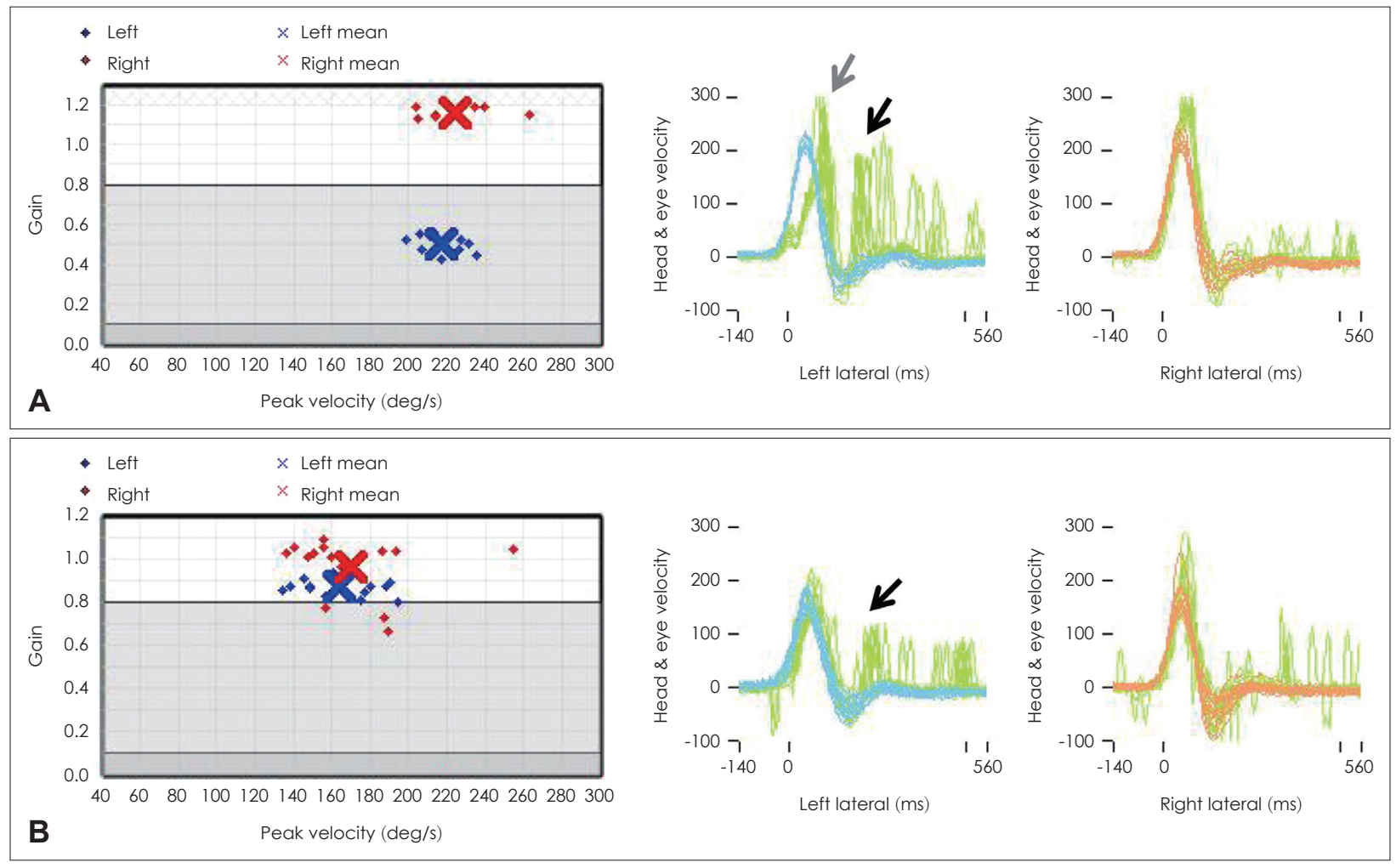

Fig. 6. Video head impulse (VHIT) test result for 55-year-old female patient diagnosed with left vestibular neuritis. In the acute stage (A), the left side gain decreased ( 0.45$)$ and definite overt (black arrow) and covert saccades (gray arrow) were visible. After 1 month (B), the gain was normalized but abnormal saccades (black arrow) remained visible on the left side. 
at 6 months after onset using vHIT. ${ }^{1,17,40)}$ However, both the gain (abnormal gain $<0.8$ ) and the saccade abnormality (abnormal saccade $>100 \%$ s) were significantly more abnormal (Fig. 6; at 1 month, 29\% when only the gain was analyzed and $13 \%$ when the saccade was analyzed with the gain). ${ }^{17,41)}$ According to Büki, et al., ${ }^{1)}$ vertical canals had a lower recovery rate than lateral canals and the lowest recovery rate was observed when only the posterior canal was involved. In contrast, Magliulo, et al. ${ }^{26)}$ reported that when vestibular function was evaluated in combination with VEMP, regions in the superior vestibular nerve showed a slower recovery of function. This finding was explained by the earlier recovery of the utricular and saccular nerves followed by the ampullary nerve. ${ }^{27)}$ When a decrease in the vHIT gain or canal paresis was severe in the acute stage, the recovery of the gain was found to be significantly lower after this stage. ${ }^{42)}$ In the study by Cerchiai, et al., ${ }^{43)}$ patients with significantly lower vHIT gain in the early stage had a significantly higher dizziness handicap index (DHI) score at 1 month and vestibular rehabilitation was necessary for these patients.

Symptom improvement during the recovery process for $\mathrm{VN}$ varies for each patient. In some cases of $\mathrm{VN}$, the symptom is long-lasting and changes in the symptoms do not always coincide with the results of the vestibular function test. ${ }^{44)}$ The rate of improvement (normalized to within 10) of DHI in VN has been reported to be approximately $64 \%$ to $87 \%{ }^{27,32)}$ However, the association between the severity of dizziness symptoms and vHIT gain has been reported differently in studies. Patel, et al. ${ }^{44)}$ found that the severity of dizziness evaluated using DHI and the Vertigo Symptoms Scale short-form in patients with chronic stage VN was not significantly correlated with the results of vHIT.

\section{Conclusion}

The vHIT is relatively simple to perform, has a short test time, and has the advantages of quantitatively evaluating each of the semicircular canals. Recently, vHIT equipment was widely adopted in many institutions in Korea. This device can be useful for differential diagnosis of patients with AUV including VN. In addition, it can be useful for AUV classification according to the extent of $\mathrm{VN}$ involvement, prediction of prognosis, and identification of treatment progress.

\section{Acknowledgments}

This work was supported by the National Research Foundation of Korea(NRF) grant funded by the Korea government(Ministry of
Science and ICT) (No. 2019R1C1C1011660).

\section{ORCID}

Myung Hoon Yoo https://orcid.org/0000-0002-9158-8924

\section{REFERENCES}

1) Büki B, Hanschek M, Jünger H. Vestibular neuritis: Involvement and long-term recovery of individual semicircular canals. Auris Nasus Larynx 2017;44(3):288-93.

2) Strupp M, Magnusson M. Acute unilateral vestibulopathy. Neurol Clin 2015;33(3):669-85.

3) Celebisoy N. Acute vestibular syndrome: Clinical head impulse test versus video head impulse test. J Neurol 2018;265(Suppl 1):44-7.

4) Guan Q, Zhang L, Hong W, Yang Y, Chen Z, Lu P, et al. Video head impulse test for early diagnosis of vestibular neuritis among acute vertigo. Can J Neurol Sci 2017;44(5):556-61.

5) MacDougall HG, Weber KP, McGarvie LA, Halmagyi GM, Curthoys IS. The video head impulse test: Diagnostic accuracy in peripheral vestibulopathy. Neurology 2009;73(14):1134-41.

6) Kattah JC, Talkad AV, Wang DZ, Hsieh YH, Newman-Toker DE. HINTS to diagnose stroke in the acute vestibular syndrome: Threestep bedside oculomotor examination more sensitive than early MRI diffusion-weighted imaging. Stroke 2009;40(11):3504-10.

7) Alhabib SF, Saliba I. Video head impulse test: A review of the literature. Eur Arch Otorhinolaryngol 2017;274(3):1215-22.

8) Choi JY, Kim HJ, Kim JS. Recent advances in head impulse test findings in central vestibular disorders. Neurology 2018;90(13): 602-12.

9) Kattah JC. Use of HINTS in the acute vestibular syndrome. An overview. Stroke Vasc Neurol 2018;3(4):190-6.

10) Chen L, Todd M, Halmagyi GM, Aw S. Head impulse gain and saccade analysis in pontine-cerebellar stroke and vestibular neuritis. Neurology 2014;83(17):1513-22.

11) Newman-Toker DE, Saber Tehrani AS, Mantokoudis G, Pula JH, Guede CI, Kerber KA, et al. Quantitative video-oculography to help diagnose stroke in acute vertigo and dizziness: Toward an ECG for the eyes. Stroke 2013;44(4):1158-61.

12) Halmagyi GM, Chen L, MacDougall HG, Weber KP, McGarvie LA, Curthoys IS. The video head impulse test. Front Neurol 2017; 8:258.

13) Mantokoudis G, Tehrani AS, Wozniak A, Eibenberger K, Kattah JC, Guede CI, et al. VOR gain by head impulse video-oculography differentiates acute vestibular neuritis from stroke. Otol Neurotol 2015;36(3):457-65.

14) Mantokoudis G, Saber Tehrani AS, Wozniak A, Eibenberger K, Kattah JC, Guede CI, et al. Impact of artifacts on VOR gain measures by video-oculography in the acute vestibular syndrome. J Vestib Res 2016;26(4):375-85.

15) Guler A, Karbek Akarca F, Eraslan C, Tarhan C, Bilgen C, Kirazli $\mathrm{T}$, et al. Clinical and video head impulse test in the diagnosis of posterior circulation stroke presenting as acute vestibular syndrome in the emergency department. J Vestib Res 2017;27(4):233-42.

16) Lee SH, Yoo MH, Park JW, Kang BC, Yang CJ, Kang WS, et al. Comparison of video head impulse test (vHIT) gains between two commercially available devices and by different gain analytical methods. Otol Neurotol 2018;39(5):e297-300.

17) Yang CJ, Cha EH, Park JW, Kang BC, Yoo MH, Kang WS, et al. Diagnostic value of gains and corrective saccades in video head impulse test in vestibular neuritis. Otolaryngol Head Neck Surg 2018;159(2):347-53.

18) Yang CJ, Lee JY, Kang BC, Lee HS, Yoo MH, Park HJ. Quantitative analysis of gains and catch-up saccades of video-head-impulse testing by age in normal subjects. Clin Otolaryngol 2016;41(5):532-8. 
19) Yoo MH, Kim SH, Lee JY, Yang CJ, Lee HS, Park HJ. Results of video head impulse and caloric tests in 36 patients with vestibular migraine and 23 patients with vestibular neuritis: A preliminary report. Clin Otolaryngol 2016;41(6):813-7.

20) The Korean Balance Society. Clinical vestibular science. Dizziness and vertigo. Seoul: Panmun Education;2017.

21) Pogson JM, Taylor RL, Young AS, McGarvie LA, Flanagan S, Halmagyi GM, et al. Vertigo with sudden hearing loss: Audiovestibular characteristics. J Neurol 2016;263(10):2086-96.

22) Yao Q, Xu C, Wang H, Shi H, Yu D. Video head impulse test results suggest that different pathomechanisms underlie sudden sensorineural hearing loss with vertigo and vestibular neuritis: Our experience in fifty-two patients. Clin Otolaryngol 2018;43(6):1621-4.

23) Martin-Sanz E, Rueda A, Esteban-Sanchez J, Yanes J, Rey-Martinez J, Sanz-Fernandez R. Vestibular restoration and adaptation in vestibular neuritis and ramsay hunt syndrome with vertigo. Otol Neurotol 2017;38(7):e203-8.

24) Walther LE, Blödow A. Ocular vestibular evoked myogenic potential to air conducted sound stimulation and video head impulse test in acute vestibular neuritis. Otol Neurotol 2013;34(6):1084-9.

25) Taylor RL, McGarvie LA, Reid N, Young AS, Halmagyi GM, Welgampola MS. Vestibular neuritis affects both superior and inferior vestibular nerves. Neurology 2016;87(16):1704-12.

26) Magliulo G, Gagliardi S, Ciniglio Appiani M, Iannella G, Re M. Vestibular neurolabyrinthitis: A follow-up study with cervical and ocular vestibular evoked myogenic potentials and the video head impulse test. Ann Otol Rhinol Laryngol 2014;123(3):162-73.

27) Magliulo G, Iannella G, Gagliardi S, Re M. A 1-year follow-up study with C-VEMPs, O-VEMPs and video head impulse testing in vestibular neuritis. Eur Arch Otorhinolaryngol 2015;272(11): 3277-81.

28) Chihara Y, Iwasaki S, Murofushi T, Yagi M, Inoue A, Fujimoto C, et al. Clinical characteristics of inferior vestibular neuritis. Acta Otolaryngol 2012;132(12):1288-94.

29) Park JS, Kim CH, Kim MB. Comparison of video head impulse test in the posterior semicircular canal plane and cervical vestibular evoked myogenic potential in patients with vestibular neuritis. Otol Neurotol 2018;39(4):e263-8.

30) Kim JS, Kim HJ. Inferior vestibular neuritis. J Neurol 2012;259(8): 1553-60.

31) Burston A, Mossman S, Mossman B, Weatherall M. Comparison of the video head impulse test with the caloric test in patients with sub-acute and chronic vestibular disorders. J Clin Neurosci 2018; 47:294-8

32) Yoo MH, Yang CJ, Kim SA, Park MJ, Ahn JH, Chung JW, et al.
Efficacy of steroid therapy based on symptomatic and functional improvement in patients with vestibular neuritis: A prospective randomized controlled trial. Eur Arch Otorhinolaryngol 2017; 274(6):2443-51.

33) Zellhuber S, Mahringer A, Rambold HA. Relation of video-headimpulse test and caloric irrigation: A study on the recovery in unilateral vestibular neuritis. Eur Arch Otorhinolaryngol 2014; 271(9):2375-83.

34) Redondo-Martínez J, Bécares-Martínez C, Orts-Alborch M, García-Callejo FJ, Pérez-Carbonell T, Marco-Algarra J. Relationship between video head impulse test (vHIT) and caloric test in patients with vestibular neuritis. Acta Otorrinolaringol Esp 2016;67(3):156-61.

35) Blödow A, Heinze M, Bloching MB, von Brevern M, Radtke A, Lempert T. Caloric stimulation and video-head impulse testing in Ménière's disease and vestibular migraine. Acta Otolaryngol 2014; 134(12):1239-44.

36) Hannigan IP, Welgampola MS, Watson SRD. Dissociation of caloric and head impulse tests: A marker of Meniere's disease. J Neurol 2019.

37) Kang WS, Lee SH, Yang CJ, Ahn JH, Chung JW, Park HJ. Vestibular function tests for vestibular migraine: Clinical implication of video head impulse and caloric tests. Front Neurol 2016;7:166.

38) Allum JHJ, Scheltinga A, Honegger F. The effect of peripheral vestibular recovery on improvements in vestibulo-ocular reflexes and balance control after acute unilateral peripheral vestibular loss. Otol Neurotol 2017;38(10):e531-8.

39) Bronstein AM, Dieterich M. Long-term clinical outcome in vestibular neuritis. Curr Opin Neurol 2019;32(1):174-80.

40) Fu W, He F, Wei D, Bai Y, Shi Y, Wang X, et al. Recovery pattern of high-frequency acceleration vestibulo-ocular reflex in unilateral vestibular neuritis: A preliminary study. Front Neurol 2019;10:85.

41) Yang CJ, Park HJ. Advantages of considering gains and corrective saccades by video head impulse for patients with vestibular neuritis. Otolaryngol Head Neck Surg 2018;159(2):400-1.

42) Bartolomeo M, Biboulet R, Pierre G, Mondain M, Uziel A, Venail F. Value of the video head impulse test in assessing vestibular deficits following vestibular neuritis. Eur Arch Otorhinolaryngol 2014; 271(4):681-8.

43) Cerchiai N, Navari E, Sellari-Franceschini S, Re C, Casani AP. Predicting the outcome after acute unilateral vestibulopathy: Analysis of vestibulo-ocular reflex gain and catch-up saccades. Otolaryngol Head Neck Surg 2018;158(3):527-33.

44) Patel M, Arshad Q, Roberts RE, Ahmad H, Bronstein AM. Chronic symptoms after vestibular neuritis and the high-velocity vestibuloocular reflex. Otol Neurotol 2016;37(2):179-84. 


\section{정답 및 해설}

답 (4)

해 설 A "nonrecurrent" laryngeal nerve may rarely occur on the right side and enters from a more lateral course. Typically, an aberrant retroesophageal subclavian artery (arteria lusoria) or other congenital malformation of the vascular rings is present.

참고 문헌: Flint PW, Haughey BH, Lund VJ, Niparko JK, Robbins KT, Thomas JR, Lesperance MM. Cummings otolaryngology head and neck surgery. 6th ed. Canada: Saunders;2015. p.1904.

The nonrecurrent RLN occurs in $0.5 \%$ to $1 \%$ of cases and is associated with a right subclavian artery takeoff from the distal aortic arch. The right subclavian in these cases follows a retroesophageal course to the right or, less commonly, between the esophagus and the trachea. 53 Henry found that the symptoms of dysphagia secondary to subclavian interaction with the esophagus (dysphagia lusoria) were not consistently present in cases of nonrecurrent RLN. When dysphagia is present, dysphagia lusoria is difficult to separate from dysphagia referable to a pathologic condition of the thyroid. Epstein suggests nonrecurrent RLN is associated with compressive symptoms in $10 \%$ of patients typically with dysphasia.

The nonrecurrent nerve is at increased risk for paralysis with one study of 31 nonrecurrent nerves at risk demonstrating a $12.9 \%$ paralysis rate.

The nonrecurrent RLN derives from the vagus as a direct medial branch in the neck and extends-depending on its level of takeoff from the vagus and generally with a downward looping course-from behind the carotid artery to the laryngeal entry point. 참고 문헌: Randolph GW. Surgery of the thyroid and parathyroid gland. 2nd ed. Philadelphia, PA: Elsevier Saunders;2012. p.311-2. 